\title{
Otimização de Retardo na Alocação de Recursos em Redes Sem Fio f-OFDM com Agregação de Subportadoras
}

\author{
Marcus V. G. Ferreira ${ }^{1}$, Flávio H. T. Vieira ${ }^{1}$ \\ ${ }^{1}$ Instituto de Informática - Universidade Federal de Goiás (UFG) \\ Alameda Palmeiras, Quadra D, Câmpus Samambaia - Goiânia - Goiás - Brasil \\ marcusferreiradinf.ufg.br, flaviodemc.ufg.br
}

\begin{abstract}
In this paper, we propose a resource allocation scheme in wireless networks that aims to optimize the user's data delay. The performance of the resource allocation algorithm is verified and compared to others in the literature using computational simulations in terms of several QoS parameters such as fairness index, throughput and loss rate. In these simulations, we also consider current and candidate techniques for next generation networks such as carrier aggregation and f-OFDM in order to show that is possible to provide higher data rate and lower latency in relation to $4 G$ networks.
\end{abstract}

Resumo. Neste artigo, propomos um esquema de alocação de recursos em redes sem fio com o objetivo de otimizar o retardo de dados dos usuários. $O$ desempenho do algoritmo de alocação de recursos é verificado e comparado com outros da literatura utilizando simulações computacionais em termos de vários parâmetros de QoS como índice de justiça, vazão e taxa de perda. Nestas simulações, consideramos também técnicas atuais e candidatas às redes da próxima geração como agregação de portadora e f-OFDM com intuito de mostrar que é possível prover maior taxa de transmissão e menor latência em relação às redes $4 G$.

\section{Introdução}

No âmbito global, a indústria celular convergiu para a tecnologia LTE (Long Term Evolution, Evolução de Longo Prazo), incluindo o LTE-A (LTE-Advanced, LTE-Avançado), como interface sem fio. Uma indústria que estava fragmentada em múltiplas tecnologias de rede sem fio agora possui um padrão, resultando em economia e padronização em termos de infraestrutura. Sendo assim, muitos pesquisadores da área propõem que a tecnologia 5G seja uma extensão dessa interface de comunicação [Olwal et al. 2016] [Rysavy 2015].

Focando em tecnologias com o objetivo de melhorar a taxa de transmissão de dados para suportar redes sem fio da próxima geração, um dos tópicos abordados neste trabalho compreende o conceito de agregação de portadora. Este é um recurso da tecnologia LTE-A que operadores desenvolvem para prover maior taxa de dados, melhor cobertura e menor latência [Rostami et al. 2015]. Este trabalho também considera a tecnologia fOFDM (filtered-Orthogonal Frequency Division Multiplexing, Multiplexação por Divisão de Frequência Ortogonal filtrada), que possibilita uma operação fragmentada do espectro e a coexistência eficiente de múltiplas sub-bandas com o objetivo de melhorar a utilização 
do espectro [Bi et al. 2017] [Wu et al. 2016]. A escolha da tecnologia f-OFDM se justifica por melhorar a taxa de transferência do sistema em $10 \%$ ao usar as bandas livres das redes LTE, além de suportar a transmissão assíncrona de diferentes usuários e ser compatível com a tecnologia MIMO (Multiple Input Multiple Output, Múltiplas Entradas Múltiplas Saídas) [Abdoli et al. 2015].

Há várias propostas de esquemas de alocação de recursos em redes sem fio [Ferreira et al. 2015] [Guan et al. 2011] [Su et al. 2012]. Em [Su et al. 2012], é proposto um algoritmo para alocação de blocos de recursos em redes LTE baseado na heurística PSO (Particle Swarm Optimization, Otimização por Enxame de Partículas), com o objetivo de maximizar a vazão total do sistema. Em [Guan et al. 2011], é proposto um algoritmo com o objetivo de garantir o critério de taxa mínima de transmissão requerida pelo usuário. Em [Ferreira et al. 2015] é proposto um algoritmo com o objetivo de minimizar o parâmetro de retardo e atender critérios de QoS (Quality of Service, Qualidade de Serviço). Estes esquemas de alocação, em adição a outros não mencionados, tem o mesmo propósito de aplicar determinados algoritmos de otimização para atender diferentes parâmetros de QoS como retardo, vazão, fairness, dentre outros.

No contexto apresentado de alocação de recursos, o retardo é tido como essencial, principalmente para aplicações em tempo real com taxa de transmissão variável e requisitos específicos de banda, como serviços de VoIP (Voice over Internet Protocol) e de videoconferência. Estes serviços estão cada vez mais em evidência a medida que cresce de forma exponencial o número de dispositivos móveis. É importante destacar também a necessidade de desenvolvimento de novas técnicas para viabilizar o atendimento dos requisitos rigorosos de QoS para as redes 5G em termos de retardo [Gupta and Jha 2015].

Neste artigo, propomos um esquema de alocação de blocos de recurso para redes sem fio considerando técnicas atuais e de próxima geração, de forma a otimizar o retardo do sistema obtendo valores para outros parâmetros de QoS compatíveis com os de outros escalonadores da literatura. Para tal, propõe-se uma variação do algoritmo proposto em [Zhou and Wunder 2007] para obtenção de retardo ótimo em um algoritmo de escalonamento de recurso, no entanto, considerando agregação de subportadoras e modulação f-OFDM.

\section{Modelo do Sistema de Transmissão}

Considere uma rede celular baseada em LTE-A com apenas um eNB (evolved Node B, nó evoluído B) consistindo de $N$ UEs (Users Equipments, Equipamentos dos Usuários) como $\alpha=\left\{\alpha_{1}, \alpha_{2}, \ldots, \alpha_{N}\right\}$. Cada usuário $\alpha_{n} \in \alpha$ pode possuir diferentes capacidades de CA (Carrier Aggregation, Agregação de Portadora) que é modelada como $\mu=\left\{\mu_{n} \mid \mu_{n} \in\right.$ $\{1,2,3,4,5\}\}_{1 x N}$, onde $\mu_{n}$ representa o número máximo de CCs ( Component Carriers, Portadoras Componente) que $\alpha_{n} \in \alpha$ pode suportar (se $\alpha_{n} \in \alpha$ é um usuário LTE Release $\left.8, \mu_{n}=1\right)$.

Todos UEs em um dado TTI (Transmission Time Interval, Intervalo de Tempo de Transmissão) competem por $M$ CCs ortogonais sem sobreposição representados por $\beta=\left\{\beta_{1}, \beta_{2}, \ldots, \beta_{M}\right\}$ onde cada $\beta_{m} \in \beta$ possui diferente número de RBs (Resource Blocks, Blocos de Recurso) e podem ser escritos como $\gamma=\left\{\gamma_{m} \mid \gamma_{m} \in\right.$ $\{6,15,25,50,75,100\}\}_{1 x M}$, onde $\gamma_{m}$ corresponde ao número de RBs em um dado $\beta_{m}$.

Assumindo que o índice MCS (Modulation and Coding Scheme, Esquema de 
Codificação e Modulação) máximo de todos UEs em diferentes RBs é modelado como [Rostami et al. 2015]:

$$
K=\left\{k_{m, p, n} \mid k_{m, p, n} \in\{0,1, \ldots, h\}\right\}_{M x P x N},
$$

onde $k_{m, p, n}$ representa o índice MCS máximo para $\alpha_{n}$ em $\beta_{m}$ no $\mathrm{RB} p$. $\mathrm{O}$ valor de $k_{m, p, n}$ depende do intervalo de qualidade do canal entre 0 e $h$. Enquanto que o valor do $h$ para uplink é 22 e para downlink é 28 e $P=\max [\gamma]$. Também é definida uma matriz de alocação de blocos de recurso sendo uma matriz binária como segue [Rostami et al. 2015]:

$$
A=\left\{a_{m, p, n} \mid a_{m, p, n} \in\{0,1\}\right\}_{M x P x N} .
$$

Representando o mapa de alocação de RBs onde $a_{m, p, n}=1$ se e apenas se o RB $p$ localizado em $\beta_{m}$ é alocado unicamente para $\alpha_{n}$ e $a_{m, p, n}=0$ caso contrário. A matriz de alocação de recursos $A$ deve satisfazer o requisito de interferência definido na equação (3), ou seja, dois ou mais UEs nao podem utilizar o mesmo RB simultaneamente [Rostami et al. 2015]:

$$
\sum_{n=1}^{N} a_{m, p, n} \leq 1 \text { para } 1 \leq p \leq P, 1 \leq m \leq M .
$$

É definida uma matriz de alocação de $\mathrm{CC}$ como uma matriz binária $M x N$ [Rostami et al. 2015] dada por:

$$
E=\left\{e_{m, n} \mid e_{m, n}=1 \leftrightarrow \sum_{p=1}^{P} a_{m, p, n} \geq 1\right\}_{M x N}
$$

onde $e_{m, n}$ representa a condição se $\beta_{m}$ é atribuído para $\alpha_{n}$ ou não. Para representar o MCS alocado para $\alpha_{n}$ em certo TTI, uma matriz de usuários é definida como segue [Rostami et al. 2015]:

$$
B=\left\{b_{m, n} \mid b_{m, n} \in\{0,1, \ldots, h\}\right\}_{M x N},
$$

onde $b_{m, n}$ representa o índice MCS alocado para $\alpha_{n}$ em $\beta_{m}$ para cada TTI. O 3GPP TS36.213 [3GPP 2012] especifica a taxa de transmissão correspondente para cada índice MCS. Neste trabalho, para representar a relação entre o índice MCS e a taxa de transmissão, é usada a notação $r=R(b)$ onde $R$ mapeia cada índice MCS para cada taxa de transmissão conforme [3GPP 2012]; em outras palavras, $r$ é a taxa de transmissão obtida para cada UE em um RB com MCS $b$. É definida entao a matriz de taxa de transmissão dos usuários como $R=\left\{r_{m, n} \mid r_{m, n}=R\left(b_{m, n}\right)\right\}_{M x N}$ ou seja, uma matriz $M x N$ onde $r_{m, n}$ apresenta a taxa de transmissão por RB para $\alpha_{n}$ em $\beta_{m}$.

O eNB é responsável por todo procedimento de admissão, escalonamento de recursos e adaptação de link. Após receber o CSI (Channel State Information, Informação 
do Estado do Canal) de todos UEs, um mapa de alocação de recursos é construído e o índice MCS é determinado pelo eNB e depois enviado para cada UE através dos canais de controle. De acordo com o índice MCS, o tipo de modulação e a taxa de codificação para cada UE em cada CC atribuído podem ser determinados.

Muitos esquemas de alocação se baseiam na maximização da taxa de vazão total do sistema com a restrição da taxa mínima de transmissão requerida por usuário. Neste caso, a função objetivo de otimização é definida como a vazão proporcionada em um dado TTI $t$, e pode ser calculada como:

$$
f^{(t)}=\sum_{m=1}^{M} \sum_{p=1}^{P} \sum_{n=1}^{N} r_{m, n}^{(t)} \times a_{m, p, n}^{(t)}
$$

onde $r_{m, n}^{(t)}$ e $a_{m, p, n}^{(t)}$ são $r_{m, n}$ e $a_{m, p, n}$ no TTI $t$ respectivamente. Sujeito a:

$$
\begin{aligned}
& \sum_{n=1}^{N} a_{m, p, n} \leq 1, \\
& \sum_{m=1}^{M} e_{m, n} \leq \mu_{n}, \\
& b_{m, n} \leq k_{m, p, n}, \\
& r_{m, n} \geq r_{m, n}^{\min },
\end{aligned}
$$

para $1 \leq p \leq P, 1 \leq m \leq M$ e $1 \leq n \leq N$. A equação (7) assegura que cada RB na rede é atribuído para no máximo um UE. A equação (8) garante que o número de CCs atribuídos para cada UE seja menor que sua capacidade de agregação. A equação (9) garante que o índice MCS para cada UE em cada CC seja menor que o índice MCS máximo suportado para cada RB atribuído em seu CC correspondente. A equação (10) garante que o escalonador atenda a taxa de transmissão mínima requerida para cada usuário.

Neste artigo, propomos que a política de escalonamento seja baseada na minimização da função objetiva do retardo do sistema, definida por:

$$
d^{(t)}=\sum_{m=1}^{M} \sum_{p=1}^{P} \sum_{n=1}^{N} \frac{q_{m, n}^{(t)}}{r_{m, n}^{(t)}} \times a_{m, p, n}^{(t)}
$$

onde $q_{m, n}^{(t)}$ denota o tamanho do buffer no TTI $t$. As restrições são as mesmas apresentadas nas equações (7), (8), (9) e (10).

\section{Modulação f-OFDM}

Para suportar a diversidade crescente de serviços futuros providos pelas redes 5G, é esperado que a largura de banda do sistema seja dividida em várias sub-bandas, onde a 
estrutura do quadro dentro de cada sub-banda possa ser configurada de acordo com o tipo individual de tráfego e a condição do canal [Abdoli et al. 2015] [Wu et al. 2016]. A forma de onda denominada f-OFDM possibilita tal fatiamento do espectro e permite a coexistência de múltiplas sub-bandas [Wu et al. 2016] [Zhang et al. 2015].

A principal idéia da forma de onda f-OFDM é reduzir a interferência entre as subbandas adjacentes, e, para tal, o sinal OFDM de banda base de cada sub-banda é filtrado por um filtro de banda limitada para suprimir a emissão fora de banda. A estrutura em cada sub-banda, incluindo o espaçamento de subportadora, o tamanho do prefixo cíclico (CP, Cyclic Prefix) e o TTI, pode ser configurada para alcançar o objetivo relacionado a cada tipo de serviço. Por exemplo, a estrutura para a sub-banda da banda larga móvel aperfeiçoada (eMBB, Enhanced Mobile Broadband) poderia almejar alta eficiência espectral, enquanto para a comunicação de ultra-confiabilidade e baixa latência (uRLLC, Ultra-Reliability and Low Latency Communication) poderia almejar baixa latência com espaçamento entre subportadora mais largo e menor TTI [Wu et al. 2016].

Outra vantagem da forma de onda f-OFDM é suportar a transmissão assíncrona multi-usuário no uplink. Através da filtragem por UE (User Equipment, Equipamento do Usuário) para suprimir a perda fora da banda, a interferência entre UEs se torna negligível. No LTE, o sinal de avanço de tempo (TA, Timing Advance) enviado para cada UE pela estação base (BS, Base Station) resulta em larga sobrecarga de sinalização, especialmente quando um grande número de usuários está presente. Introduzindo a filtragem por UE para suprimir a interferência entre UE, as UEs não necessitam manter uma sincronização rigorosa com a BS para explorar a ortogonalidade do OFDM, e a sobrecarga de sinalização de avanço de tempo pode ser reduzida [Abdoli et al. 2015].

Nas especificações do LTE, $10 \%$ da largura de banda do sistema está reservada como banda de guarda em uma portadora base, para suportar a taxa de perda de canal adjacente (ACLR, Adjacent Channel Leakage Ration) e o requisito de espectro [Wu et al. 2016]. Aplicando f-OFDM, o sinal OFDM de banda base pode ser configurado com uma região de transição ultra estreita, e assim a banda de guarda pode ser reutilizada para transmitir sinais de dados, resultando em uma utilização eficiente do espectro [Zhang et al. 2015].

\section{Alocação de Recursos com Otimização de Retardo}

Considerando inicialmente o problema de otimização para um canal estático $h$ e o status inicial do buffer $q(t=1)$. Denota-se a taxa de chegada média como $\bar{a}$ e assume-se que não há pacotes chegando após $t=0$. Posteriormente, escolhemos o tamanho da janela de observação $T$ com $q_{n}(T)=0, \forall n \in N$ de modo que os buffers estejam completamente desocupados dentro da janela de tempo. Portanto, o esquema de escalonamento de retardo ótimo pode ser escrito como a solução do seguinte problema de otimização [Zhou and Wunder 2007]:

$$
\min \sum_{n=1}^{N} d_{n}=\min \sum_{t=1}^{T} \sum_{n=1}^{N} \frac{q_{n}^{t}}{\bar{a}_{n}},
$$

sujeito a: 


$$
\begin{gathered}
q_{n}^{t+1}=q_{n}^{t}-r_{n}^{t}, \\
r^{t} \in C(h, \bar{W}), \\
q_{n}^{t}-r_{n}^{t} \geq 0, \forall n \in N, t \in[1, \ldots, T],
\end{gathered}
$$

onde $q_{n}^{t}$ e $r_{n}^{t}$ denotam o tamanho da fila e a taxa de transmissão do usuário $n$ no tempo $t$ e $C(h, \bar{W})$ denota a região de capacidade instantânea do sistema, em função da potência do canal $\bar{W}$ e do atual estado de desvanecimento do canal $h$. Estendendo o problema (12) para cada estado de fila $q^{t}$, temos o problema de otimização equivalente [Zhou and Wunder 2007]:

$$
\min \sum_{t=1}^{T}\left(\sum_{n=1}^{N} \frac{q_{n}^{1}}{\bar{a}_{n}}-\sum_{n=1}^{N}(T-t) \frac{r_{n}^{t}}{\bar{a}_{n}}\right)
$$

sujeito a:

$$
\begin{gathered}
r^{t} \in C(h, \bar{W}), \\
q_{n}^{1}-\sum_{u=1}^{t} r_{n}^{u} \geq 0, \forall n \in N, t \in[1, \ldots, T] .
\end{gathered}
$$

A função de Langrage é [Bertsekas 1995]:

$$
L\left(r^{t}, \lambda^{t}\right)=\sum_{t=1}^{T} \sum_{n=1}^{N} \frac{q_{n}^{1}}{\bar{a}_{n}}-\sum_{t=1}^{T} \sum_{n=1}^{N}(T-t) \frac{r_{n}^{t}}{\bar{a}_{n}}-\sum_{t=1}^{T} \sum_{n=1}^{N} \lambda_{n}^{t}\left(q_{n}^{1}-\sum_{u}^{t} r_{n}^{u}\right)
$$

Denotando $\eta_{n}^{*}=T-\bar{a}_{n} \sum_{u=1}^{T} \lambda_{n}^{u}$, obtemos o $\mu_{n}^{t}$ ótimo:

$$
\mu_{n}^{t}=\left\{\begin{array}{cc}
\frac{\eta_{n}^{*}-t+1}{\bar{a}_{n}}, & n \leq \eta^{*} \\
0, & n>\eta^{*}
\end{array}\right.
$$

e o problema de otimização do retardo se transforma em:

$$
\max \sum_{t=1}^{T} \sum_{n=1}^{N} \mu^{t \top} \cdot r
$$

sujeito a:

$$
r^{t} \in C(h, \bar{W})
$$


que pode ser resolvido facilmente.

O parâmetro $\eta_{n}^{*}$ na equação (20) pode ser obtido através de aproximação iterativa descrita no Algoritmo 1 apresentado abaixo, onde $\lceil\eta\rceil$ denota o menor inteiro maior que $\eta$ e $\epsilon$ é o erro tolerável predefinido de $\eta$. $\mathrm{O} \eta^{(u)}$ obtido no Algoritmo 1 converge para $\eta^{*}$ que resulta no $\mu_{n}^{t}$ ótimo para o problema de otimização do retardo descrito na equação (12), conforme demonstrado em [Zhou and Wunder 2007].

1 Define $\mu_{n}^{(0)}=\frac{1}{\bar{a}_{n}}$ e calcula $r^{(0)}=\arg \max _{r \in C_{h}} \mu^{(0) \top} \cdot r$;

2 Inicializa o tamanho do estado não ocioso $\eta_{n}^{(0)}=\min _{n \in N} \frac{q_{n}^{1}}{r_{n}^{(0)}}$;

3 Define a ordem $\pi$ de modo que $\frac{q_{\pi(1)}^{1}}{r_{\pi(1)}^{(0)}} \geq \frac{q_{\pi(2)}^{1}}{r_{\pi(2)}^{(0)}} \geq \ldots \geq \frac{q_{\pi(N)}^{1}}{r_{\pi(N)}^{(0)}} ;$

4 Define $t=0$;

5 repita

6 Define $\eta^{(u+1)}=\eta^{(u)}$;

$7 \quad$ para $n=1$ até $N$ faça

$8 \quad \eta^{*}=\eta^{(u+1)}$;

$9 \quad$ repita

$10 \quad$ Aumenta $\eta_{\pi(n)}^{*}$. Resolva o problema de maximização descrito na equação (21) e calcula a evolução do estado da fila ;

$11 \quad \begin{aligned} \text { se } q_{\pi(n)}^{\left\lceil\eta_{\pi(n)}^{*}\right\rceil} & \geq 0 \text { então } \\ \eta_{\pi(n)}^{(u+1)} & =\eta_{\pi(n)}^{*}\end{aligned}$

$13 \quad$ fim

$14 \quad$ até $q_{\pi(n)}^{\left\lceil\eta_{\pi(n)}^{*}\right\rceil}<0$;

15 fim

$16 \quad u=u+1$;

17 até $\eta_{n}^{(u)}-\eta_{n}^{(u-1)}<\epsilon, \forall n \in N$;

$18 \eta^{*}=\eta^{(u)}$.

Algoritmo 1: Predição do Estado Ocioso

É importante notar que o Algoritmo 1 pode também ser usado caso o estado do canal $h$ varie no decorrer do tempo e a estação base tenha informação antecipada do estado de cada canal. Entretanto, a estação base possui apenas a informação do atual estado do canal e o conhecimento estatístico deste. Além disso, o processo de chegada de pacotes não é ergódico e não pode ser previsto. De forma a evitar o possível retardo de fila infinito, a política de retardo ótimo pode também ser ótima em termos de vazão, e assim o estado da fila se mantém estável para qualquer taxa de chegada esperada $\rho$ dentro da região de capacidade ergódica.

Se nenhum novo pacote chega após o slot de tempo $t=0$, o retardo esperado para uma dada política $\mathbf{P}$ é [Zhou and Wunder 2007]:

$$
\mathbf{E}\left\{\sum_{t=1}^{T} \sum_{n=1}^{N} d_{n}^{t}\right\}=\mathbf{E}\left\{\sum_{t=1}^{T}\left(\sum_{n=1}^{N} \frac{q_{n}^{1}}{\bar{a}_{n}}-\sum_{n=1}^{N}(T-t) \frac{r_{n}^{\mathbf{P} t}}{\bar{a}_{n}}\right)\right\},
$$


onde $r_{n}^{\mathbf{P} t}$ é a taxa alocada pela política $\mathbf{P}$ para o $n$-ésimo usuário no slot de tempo $t$. Se $\mathbf{P}$ é uma política de vazão ótima, então:

$$
\mathbf{E}\left\{r^{P}\right\}=\arg \max _{r \in C_{e r g}(\bar{w})}\left(\mu^{\mathbf{P}}\right)^{\top} \cdot r
$$

onde $\mu^{\mathbf{P}}$ independe do atual estado do canal. Consequentemente, o problema de otimização é equivalente a:

$$
\min \sum_{t=1}^{T}\left(\sum_{n=1}^{N} \frac{q_{n}^{1}}{\bar{a}_{n}}-\sum_{n=1}^{N}(T-t) \frac{\tilde{r}_{n}^{t}}{\bar{a}_{n}}\right)
$$

sujeito a:

$$
\begin{gathered}
\tilde{r}^{t} \in C_{e r g}(\bar{W}), \\
q_{n}^{1}-\sum_{u=1}^{t} r_{n}^{u} \geq 0, \forall n \in N, t \in[1, \ldots, T],
\end{gathered}
$$

onde $C_{e r g}(\bar{W})$ é a região de capacidade ergódica do canal.

Assim, o problema de otimização pode ser resolvido utilizando o Algoritmo 2 [Zhou and Wunder 2007].

1 para cada slot de tempo $t$ faça

2 Calcula a taxa de chegada média anterior $\bar{a}$;

$3 \quad$ Calcula $\eta^{*}$ de acordo com $\bar{a}$ e o atual estado da fila $q$ usando Algoritmo $\mathbf{1}$, onde a região de canal estática $C(h, \bar{W})$ é substituída pela região de capacidade ergódica $C_{\text {erg }}(\bar{W})$;

$4 \quad$ Calcula o vetor de peso atual $\tilde{\mu}^{1}$ de acordo com a equação (20);

5 Calcula a taxa atual de alocação:

$$
r^{*}=\arg \max _{r \in C\left(h^{t}, \bar{w}\right)}\left(\tilde{\mu}^{1}\right)^{\top} \cdot r
$$

6 fim

onde $h^{t}$ é o atual estado do canal.

Algoritmo 2: Escalonamento para Otimização de Retardo

No sistema com nova chegada de pacotes, o vetor de pesos $\tilde{\mu}^{1}$ deve ser recalculado conforme o novo estado da fila e a taxa de alocação é determinada com $\tilde{\mu}^{1}$ e o atual estado do canal $h$.

Propomos aplicar o Algoritmo 2 na alocação de recursos em redes sem fio de forma a considerar agregação de subportadoras e modulação f-OFDM. O uso dessas técnicas influencia a capacidade do canal no Algoritmo 2 de acordo com a equação (26) e 
consequentemente a taxa de transmissão final para os usuários. Dessa forma, pretende-se melhorar o desempenho da rede em termos de retardo de dados dos usuários.

O algoritmo proposto de alocação de recursos acrescenta ao processo de otimização uma parte dedicada à minimização de retardo com base no comportamento do tamanho da fila no buffer do usuário, diferente dos outros algoritmos considerados. De fato, os outros algoritmos considerados neste trabalho estão voltados a maximizar a vazão total da rede, porém sem considerar predição de estado ocioso ou otimização específica do retardo.

\section{Simulações e Resultados}

Nesta seção são apresentados os resultados das simulações do algoritmo proposto de alocação de blocos de recurso em comparação a outros algoritmos da literatura, implementados através do software MATLAB versão R2015a. Foram considerados em simulação o mesmo cenário de uma rede sem fio para todos os algoritmos com agregação de subportadoras e modulação f-OFDM, além da mesma modelagem de canal e condição de tráfego.

As condições de canal para cada usuário e RBs em termos de SINR (Signal-toInterference-plus-Noise-Ratio, Relação Sinal Ruído mais Interferência) foram geradas para cada TTI conforme os parâmetros apresentados na Tabela 1. Os valores descritos são similares aos valores encontrados nas referências [Ni et al. 2013] [3GPP 2008] [3GPP 2011].

\begin{tabular}{|l|l|}
\hline Modelo multipercurso & Rayleigh \\
\hline Perfil de atraso multipercurso & $\begin{array}{l}\text { ETU (Extended Typical Urban, } \\
\text { Urbano Típico Estendido) }\end{array}$ \\
\hline Modelo de perda de percurso & $\begin{array}{l}L=128.1+37.6 \log 10(R), R \text { em } \\
\text { quilômetro }\end{array}$ \\
\hline Sombreamento lognormal & Média 0 e desvio padrão 10dB \\
\hline Distância entre UE e eNB & $1 \mathrm{~km}$ \\
\hline Densidade de potência do ruído branco & $-174 \mathrm{dBm} / \mathrm{Hz}$ \\
\hline Potência máxima de transmissão do eNB & $46 \mathrm{dBm}$ \\
\hline Ganho da antena do eNB após perda do cabo & $15 \mathrm{dBi}$ \\
\hline Ganho da antena do UE & $0 \mathrm{dBi}$ \\
\hline Figura de ruído do UE & $9 \mathrm{~dB}$ \\
\hline Margem de interferência do UE & $4 \mathrm{~dB}$ \\
\hline Velocidade do UE & $3 \mathrm{~km} / \mathrm{h}$ \\
\hline
\end{tabular}

Tabela 1. Parâmetros de simulação para modelagem de canal

As simulações foram desenvolvidas considerando os parâmetros para um cenário de transmissão downlink apresentado na Tabela 2, similares aos valores encontrados nas referências [Ferreira et al. 2015] [Guan et al. 2011] [Su et al. 2012]. A taxa de bit e o índice MCS associado ao SINR são definidos com CQI (Channel Quality Indicator, Indicador de Qualidade de Canal) de 4 bits conforme [Kawser et al. 2012]. A heurística PSO foi implementada utilizando 30 indivíduos e 100 iterações máximas como critério de parada. 


\begin{tabular}{|l|l|}
\hline Número de blocos de recurso por portadora & {$[255050100100]$} \\
\hline Taxa de transmissão mínima requerida por usuário & $0.768 \mathrm{Mbits} / \mathrm{s}$ \\
\hline Tamanho do subframe & $1 \mathrm{~ms}$ \\
\hline Número de TTIs simulados & 1000 \\
\hline Forma de onda & f-OFDM \\
\hline
\end{tabular}

Tabela 2. Cenário de simulação da transmissão downlink

As simulações foram desenvolvidas comparando os resultados do algoritmo proposto com os seguintes algoritmos de alocação de recursos: algoritmo baseado em PSO [Su et al. 2012], algoritmo QoS guaranteed [Guan et al. 2011] e algoritmo de minimização de retardo, denominado Min-delay [Ferreira et al. 2015]. Os valores médios apresentados nos resultados podem ser confirmados com forte certeza, uma vez que correspondem à media de 1000 realizações dos algoritmos, onde podem ser facilmente inseridos os intervalos de confiança para as curvas.

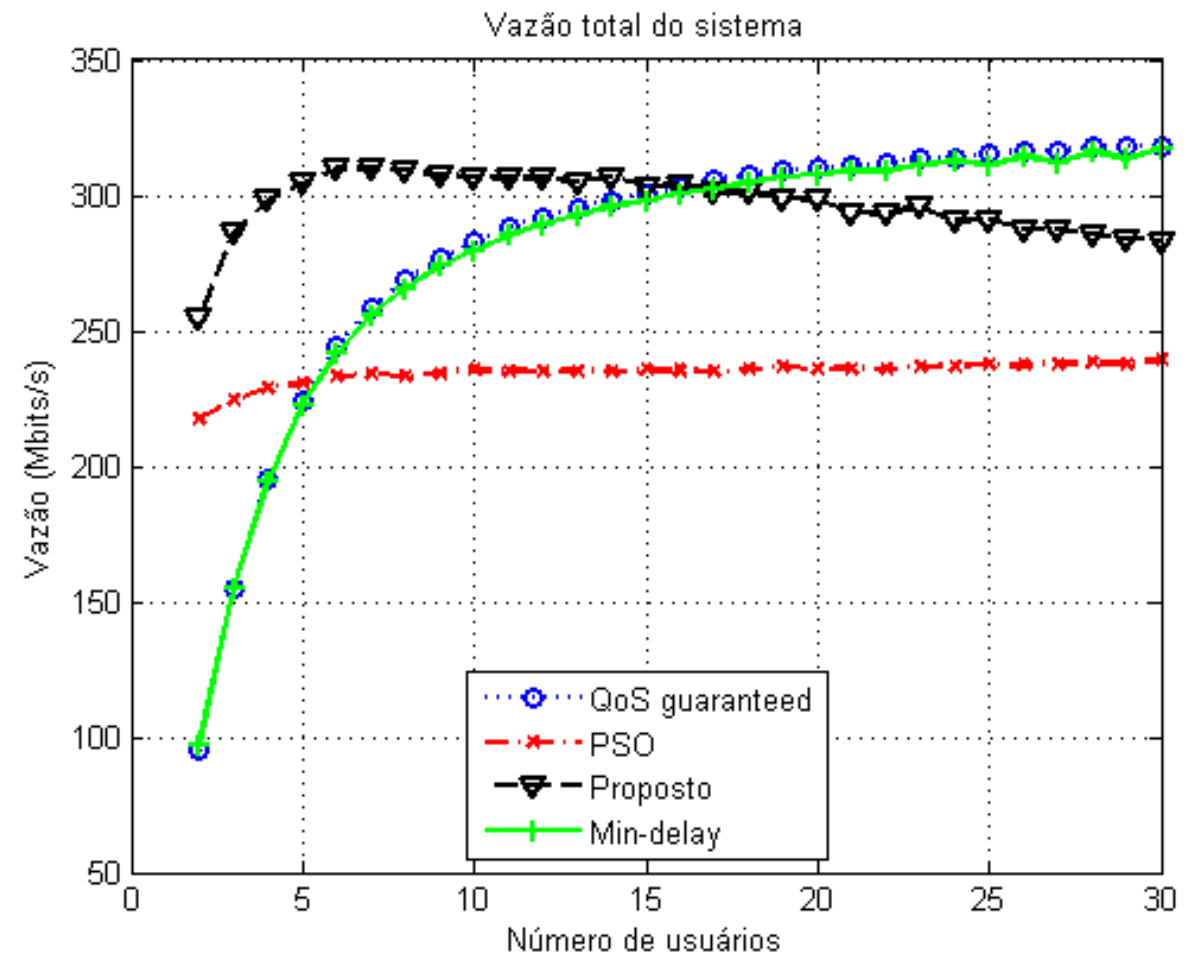

Figura 1. Vazão total utilizando f-OFDM

A Figura 1 mostra que o algoritmo proposto apresenta melhor desempenho em termos de vazão total se considerado até 15 usuários na alocação. O fato se justifica pela característica do algoritmo proposto de otimizar o parâmetro de retardo e vazão para certas situações, conforme apresentado na Seção 4. O algoritmo PSO apresenta pior desempenho no geral, devido a sua função de penalidade.

Em termos de vazão média, a Figura 2 mostra que o algoritmo proposto apresenta o melhor desempenho para todos os cenários. A partir de 25 usuários considerados em simulação, os algoritmos tendem a apresentar valores similares de vazão média. 


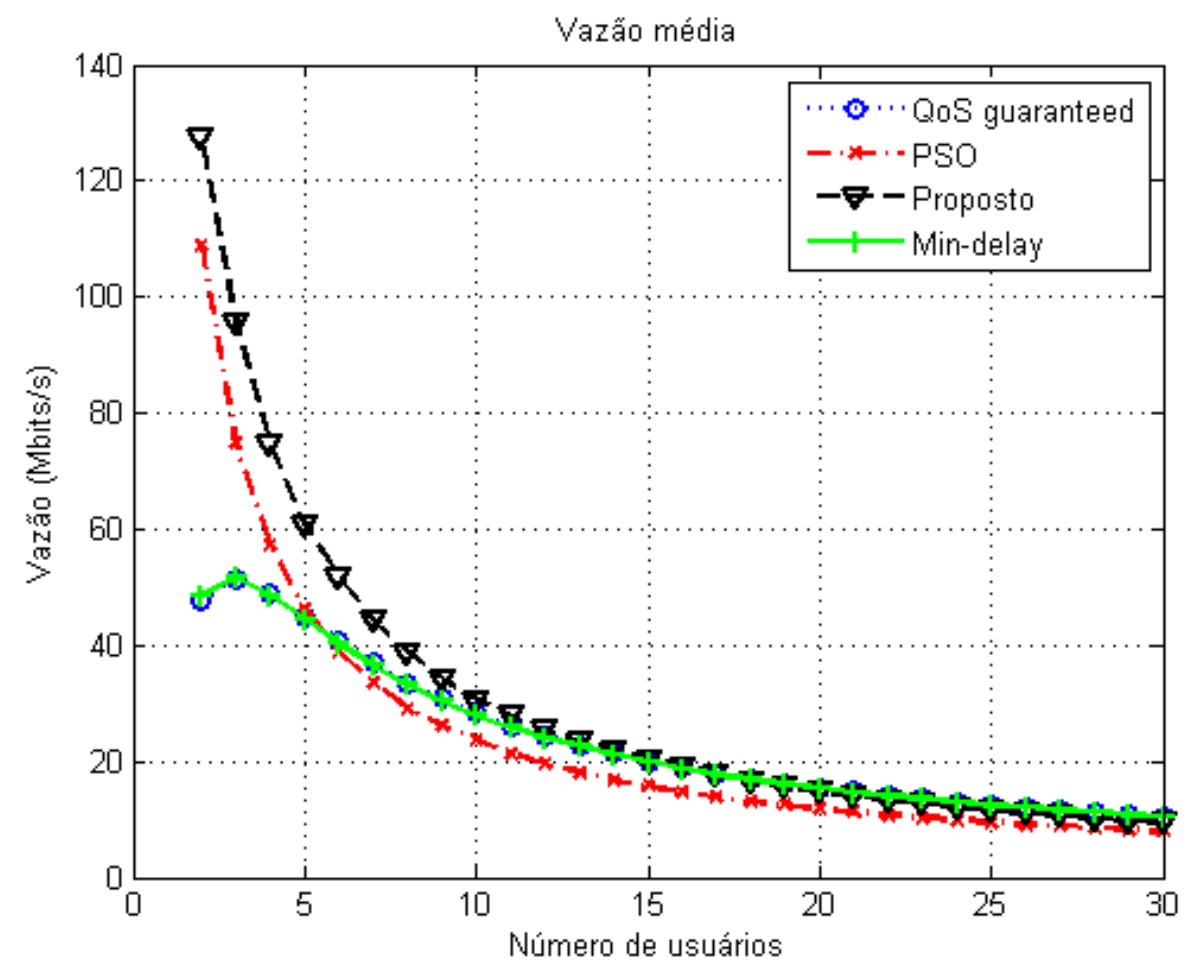

Figura 2. Vazão média utilizando f-OFDM

O algoritmo proposto apresenta os menores valores de retardo para todos os cenários simulados com diferente número de usuários, conforme mostrado na Figura 3, comprovando que o Algoritmo 2 é efetivo em sua proposta de otimizar o retardo.

A Figura 4 mostra que o algoritmo proposto apresenta valores de fairness (índice de justiça) acima de 0.85 , ou seja, a distribuição dos recursos entre os usuários é realizada de forma justa. $\mathrm{O}$ algoritmo PSO apresenta o pior desempenho no geral.

Com relação aos dados da Figura 5, esta se refere à perda média de bits no sistema considerado. Analisando este parâmetro, verifica-se que o algoritmo proposto apresenta valores próximos de zero, menor que todos os demais algoritmos para todos os cenários simulados. O algoritmo PSO apresenta pior desempenho em termos de taxa de perda.

\section{Conclusão}

Propomos neste artigo um esquema de alocação de blocos de recurso para redes sem fio, utilizando técnicas atuais e da próxima geração, que objetiva otimizar o retardo de dados do usuário, mantendo elevados níveis de vazão, conforme apresentado na Seção 4. Mostramos que a política de escalonamento pode ser formulada como um problema de maximização da vazão total e que a escolha dos pesos atribuídos, conforme equação (21), tem impacto na otimização do retardo.

A inovação deste trabalho em relação a [Zhou and Wunder 2007] se dá por estendermos o algoritmo de alocação de recursos para o cenário com agregação de subportadoras. Além disso, também se diferencia por considerar um sistema com modulação f-OFDM, comparando seu desempenho com outros algoritmos da literatura. De fato, 


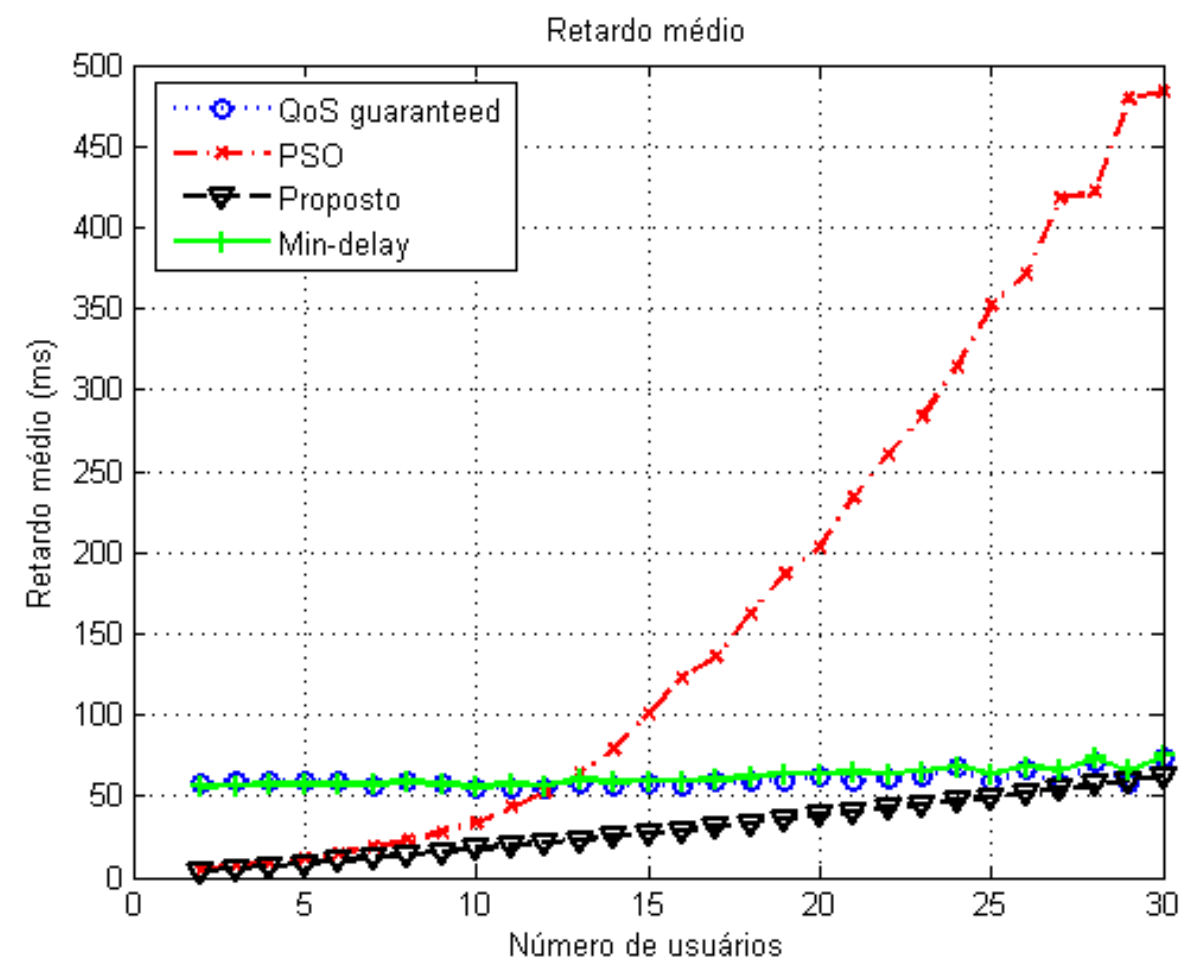

Figura 3. Retardo utilizando f-OFDM

propomos uma heurística de alocação de recursos que demanda menor capacidade de processamento em relação a um algoritmo baseado em enxame de partículas, mas com desempenho geral superior, conforme constatado via simulações.

Verifica-se através dos resultados apresentados na Seção 5 que o algoritmo proposto de alocação de recursos com minimização de retardo apresenta melhor desempenho em termos de retardo e taxa de perda para todos os cenários simulados com diferente número de usuários, quando comparado com os algoritmos PSO, QoS Guaranteed e Mindelay. O algoritmo proposto também apresenta valores de vazão total maiores que os demais algoritmos quando considerado até 15 usuários em simulação e valores de vazão média maiores que os demais algoritmos. Estes resultados comprovam que o algoritmo proposto na Seção 4 é eficiente em sua proposta de otimização de retardo.

\section{Referências}

3GPP (2008). 3gpp ts 36.104 version 8.3.0 release 8. 1te; evolved universal terrestrial radio access (e-utra); base station (bs) radio transmission and reception.

3GPP (2011). 3gpp tr 36.931 version 9.0.0 release 9. lte; evolved universal terrestrial radio access (e-utra); radio frequency (rf) requirements for lte pico node $b$.

3GPP (2012). 3gpp ts 36.213 version 10.4.0 release 10. 1te; evolved universal terrestrial radio access (e-utra); physical layer procedures.

Abdoli, J., Jia, M., and Ma, J. (2015). Filtered ofdm: A new waveform for future wireless systems. In 2015 IEEE 16th International Workshop on Signal Processing Advances in Wireless Communications (SPAWC), pages 66-70. 


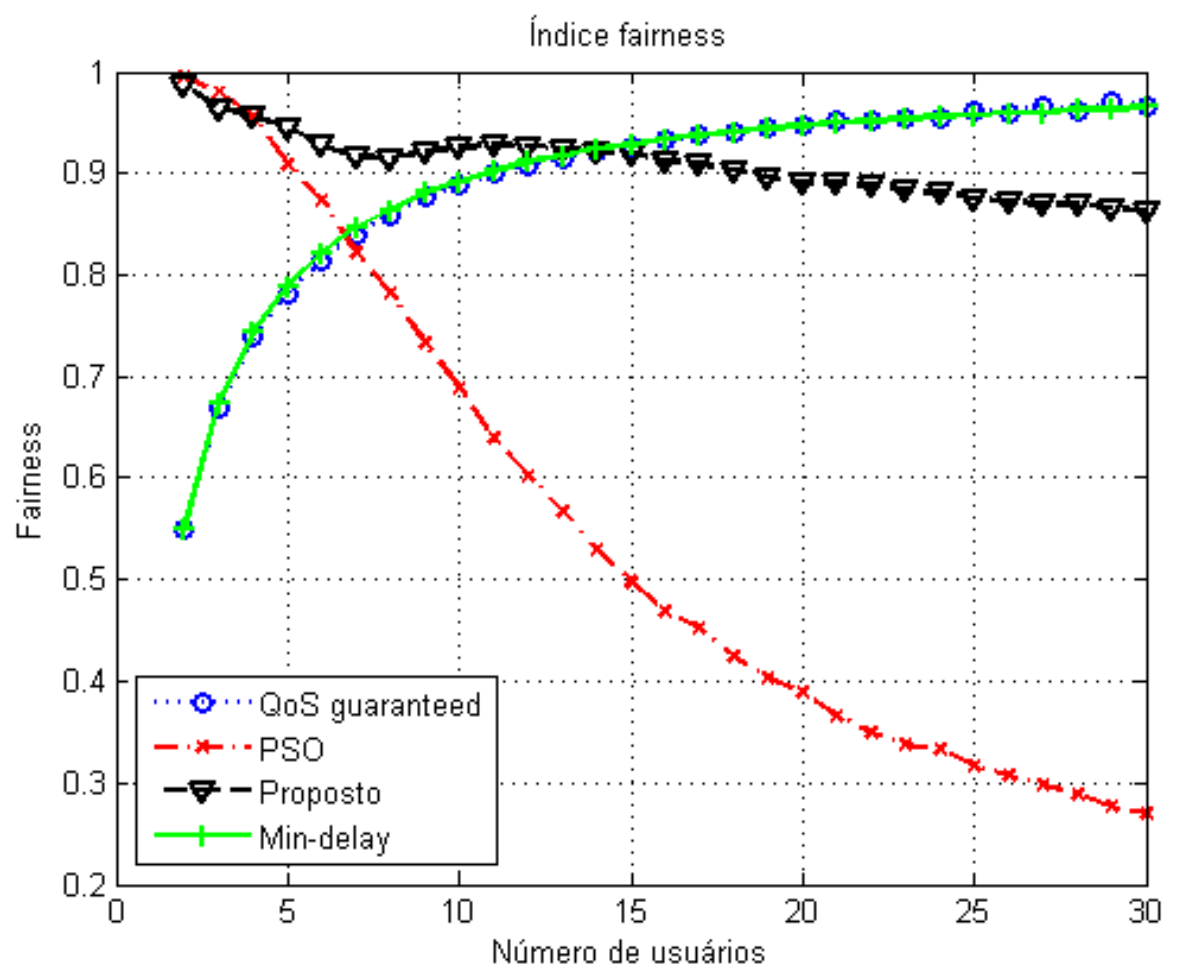

Figura 4. Índice fairness utilizando f-OFDM

Bertsekas, D. (1995). Nonlinear Programming. Athena Scientific.

Bi, M., Jia, W., Li, L., Miao, X., and Hu, W. (2017). Investigation of f-ofdm in 5g fronthaul networks for seamless carrier-aggregation and asynchronous transmission. In 2017 Optical Fiber Communications Conference and Exhibition (OFC), pages 1-3.

Ferreira, M. V. G., Vieira, F. H. T., and Abrahão, D. C. (2015). Minimizing delay in resource block allocation algorithm of lte downlink. In 2015 International Workshop on Telecommunications (IWT), pages 1-7.

Guan, N., Zhou, Y., Tian, L., Sun, G., and Shi, J. (2011). Qos guaranteed resource block allocation algorithm for lte systems. In 2011 IEEE 7th International Conference on Wireless and Mobile Computing, Networking and Communications (WiMob), pages 307-312.

Gupta, A. and Jha, R. K. (2015). A survey of 5g network: Architecture and emerging technologies. IEEE Access, 3:1206-1232.

Kawser, M., Imtiaz Bin Hamid, N., Nayeemul Hasan, M., Shah Alam, M., and Musfiqur Rahman, M. (2012). Downlink snr to cqi mapping for different multiple antenna techniques in lte. 2:756-760.

Ni, M., Xu, X., and Mathar, R. (2013). A channel feedback model with robust sinr prediction for lte systems. In 2013 7th European Conference on Antennas and Propagation (EuCAP), pages 1866-1870.

Olwal, T. O., Djouani, K., and Kurien, A. M. (2016). A survey of resource management toward $5 \mathrm{~g}$ radio access networks. IEEE Communications Surveys Tutorials, 


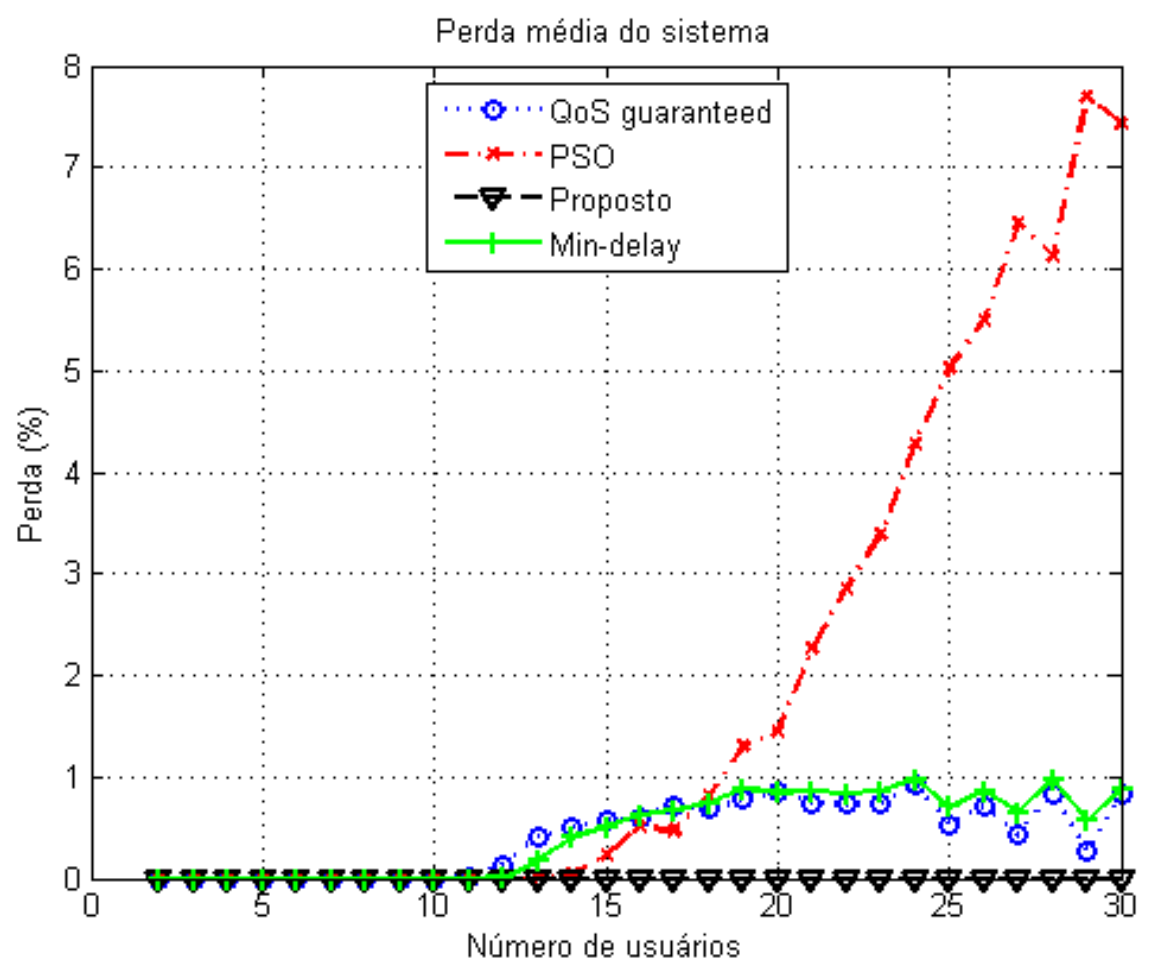

Figura 5. Taxa de perda utilizando f-OFDM

18(3):1656-1686.

Rostami, S., Arshad, K., and Rapajic, P. (2015). A joint resource allocation and link adaptation algorithm with carrier aggregation for $5 \mathrm{~g}$ lte-advanced network. In 2015 22nd International Conference on Telecommunications (ICT), pages 102-106.

Rysavy (2015). LTE and 5G Innovation: Igniting Mobile Broadband. Rysavy Rese$\operatorname{arch} / 4 \mathrm{G}$ Americas.

Su, L., Wang, P., and Liu, F. (2012). Particle swarm optimization based resource block allocation algorithm for downlink lte systems. In 2012 18th Asia-Pacific Conference on Communications (APCC), pages 970-974.

Wu, D., Zhang, X., Qiu, J., Gu, L., Saito, Y., Benjebbour, A., and Kishiyama, Y. (2016). A field trial of f-ofdm toward 5g. In 2016 IEEE Globecom Workshops (GC Wkshps), pages $1-6$.

Zhang, X., Jia, M., Chen, L., Ma, J., and Qiu, J. (2015). Filtered-ofdm - enabler for flexible waveform in the 5th generation cellular networks. In 2015 IEEE Global Communications Conference (GLOBECOM), pages 1-6.

Zhou, C. and Wunder, G. (2007). Delay and throughput optimal scheduling for ofdm broadcast channels. In Proc. IEEE Globecom 2007. IEEE International Symposium on Information Theory (ISIT2007). 